\title{
isions
}

Revista Educación

ISSN: 0379-7082

ISSN: 2215-2644

revedu@gmail.com

Universidad de Costa Rica

Costa Rica

\section{Realidades y desafíos de la educación intercultural bilingüe y la interculturalidad: el caso de las personas afrocostarricenses}

Bell Jiménez, Ana Gabriela

Realidades y desafíos de la educación intercultural bilingüe y la interculturalidad: el caso de las personas afrocostarricenses

Revista Educación, vol. 44, núm. 2, 2020

Universidad de Costa Rica, Costa Rica

Disponible en: http://www.redalyc.org/articulo.oa? $\mathrm{id}=44062184020$

DOI: https://doi.org/10.15517/revedu.v44i2.39338

Esta obra está bajo una Licencia Creative Commons Atribución-NoComercial-SinDerivar 3.0 Internacional. 


\title{
Realidades y desafíos de la educación intercultural bilingüe y la interculturalidad: el caso de las personas afrocostarricenses
}

\author{
Facts and Challenges Facing Bilingual Intercultural Education and Interculturalism: A Case Study of Afro-Costa \\ Ricans
}

Ana Gabriela Bell Jiménez

Universidad Estatal a Distancia, Costa Rica

gabibelljimenez@gmail.com

iD http://orcid.org/0000-0002-7470-8947
DOI: https://doi.org/10.15517/revedu.v44i2.39338

Redalyc: http://www.redalyc.org/articulo.oa?id=44062184020

Recepción: 14 Noviembre 2019

Aprobación: 13 Mayo 2020

\section{Resumen:}

Este ensayo argumenta que la educación intercultural bilingüe y la interculturalidad no han logrado sus propósitos para las personas afrocostarricenses. Con este fin, profundizo en los conceptos de educación intercultural bilingüe e interculturalidad, por medio de un referente histórico y actual anclado en aristas sociológicas, antropológicas y pedagógicas. La metodología de construcción del ensayo constó de interpretación y análisis de contenidos de diversos documentos académicos, históricos y jurídicos inherentes a los conceptos. Además, entrevisté a actores políticos de la educación intercultural costarricense. En la última sección planteo las posibilidades de una educación en vista a la interculturalidad y el bilingüismo por y para afrocostarricenses.

Palabras Clave: Educación intercultural bilingüe, Interculturalidad, Poblaciones marginadas, Afrocostarricenses, Afrodescendientes.

\section{Abstract:}

This essay argues that Intercultural Bilingual Education (IBE) and interculturality have not yet fulfilled their intended goal with regards to Afro-Costa Ricans. This study delves into the concepts of IBE and interculturality based on both historical and current reference points underpinned by sociological, anthropological and pedagogical perspectives. The methodology used for this study was based on analysis of historical, academic and legal documents as well an interview conducted with a leading Costa Rican Intercultural Education policy-maker. The last section considers an intercultural and bilingual educational program by and for Afro-Costa Ricans.

KEYWORDS: Intercultural Bilingual Education, Interculturality, Marginalized Populations, Afro-Costa Ricans, Afrodescendant.

\section{INTRODUCCIÓN}

Los sistemas educativos son el semillero de la sociedad en que vivimos. Por medio de estos, en cada contexto socio histórico en el que se educa, se repiten patrones sociopolíticos locales, regionales o nacionales (Suasnábar, 2013). La educación tiene un papel importante como agente catalizador para el cambio o el mantenimiento social. Así entendida, la temática educativa con poblaciones históricamente marginadas por su diversidad lingüística, étnica, racial y cultural, es compleja.

En Costa Rica, como en el resto de Iberoamérica, la diversidad cultural está constituida por cientos de poblaciones aborígenes y afrodescendientes. Esta diversidad ha sido reconocida a nivel político en diversas cumbres presidenciales o reuniones de jefes de Estado. En algunos países se han creado leyes para reconocer la pluriculturalidad (García y Martinell, 2010).

De no existir un discurso nacional, la discriminación y los conflictos de índole étnica continúan siendo la norma. En la actualidad varios estudios han intentado clarificar entre lo que nos diferencia y lo que nos aproxima como pueblo Iberoamericano. Las poblaciones afrodescendientes se estiman tres veces más que las 
indígenas, tienen territorios poco definidos, suelen vivir bajo índices de pobreza, tienen poca organización política y por ende bajo reconocimiento del Estado (García y Martinell, 2010).

Este ensayo tiene tres objetivos:

1. Profundizar en los conceptos educación intercultural bilingüe e interculturalidad.

2. Exponer un referente histórico y actual de las personas afrocostarricenses a partir de la educación intercultural bilingüe y la interculturalidad en Costa Rica.

3. Argumentar que la educación intercultural bilingüe y la interculturalidad en Costa Rica no han logrado sus propósitos para las personas afrocostarricenses.

Este documento hace un aporte al área de estudio, particularmente al estado del arte desde la vivencia de las personas afrocostarricenses en el contexto de la interculturalidad en América Latina.

\section{Metodología}

Los insumos utilizados para este ensayo provienen de dos fuentes. La primera, una rigurosa revisión documental (Suárez, 2007; Bolseguí y Fuguet, 2006; Cisterna, 2005) la cual empezó con un arqueo general, búsquedas sistémicas y delimitación de fuentes; y fue seguida por la sistematización, el análisis y la interpretación de contenidos antropológicos (Harro, 2000; Bonfil, 1991; Greene, 2007; Molina, 2015), sociológicos (Maldonado, 2007; Pierce, 1995; Quijano, 2000; Suasnábar, 2013; Walsh, 2009, 2014; Duncan, 2005), y pedagógicos (Corbetta, Bonetti, Bustamante y Vergara, 2018; Dietz, 2012; Pizarro, 2003; Walsh 2008). Las categorías de análisis surgieron de las primeras dos preguntas de investigación: (a) los conceptos de educación intercultural bilingüe e interculturalidad y (b) referentes históricos y actuales de las personas afrocostarricenses a partir de la educación intercultural bilingüe y la interculturalidad en Costa Rica. Un ficheo textual me permitió la identificación de subcategorías con el fin de crear una propuesta teórica validada en la triangulación de conocimientos. La segunda, una entrevista a actores políticos de la educación intercultural bilingüe en Costa Rica. La entrevista estructurada de tres preguntas se dirigió a personas funcionarias del Departamento de Educación Intercultural del Ministerio de Educación Pública por medio de una comunicación personal al buzón oficial del departamento (2019). Utilicé un proceso de codificación abierta (Fernández, 2006; Flick, 2007) a nivel de párrafo de la cuál surgieron códigos en vivo, tomados de las expresiones de los entrevistados. De ambas fuentes, seleccioné los contenidos que discutieran o explicaran exitosamente los conceptos-temas identificados o aportaran evidencia de la realidad histórica o actual de las personas afrocostarricenses. Apliqué un proceso de codificación axial (Fernández, 2006; Flick, 2007) para el análisis e integración de la información con el fin de relacionar subcategorías con categorías de los códigos desarrollados. Posteriormente, identifiqué las categorías más relevantes a las preguntas de investigación. Para fundamentar las conclusiones interpreté y sinteticé las ideas expuestas en vista a la base teórica para identificar condiciones causales o explicativas entre el fenómeno y el contexto, entre las condiciones interpuestas y sus consecuencias.

\section{LA EDUCACIÓN INTERCULTURAL BILINGÜE Y LA INTERCULTURALIDAD.}

La educación intercultural bilingüe es la modalidad educativa identificada por las Naciones Unidas, que tiene como fin atender la educación de pueblos indígenas y poblaciones afrodescendientes en América Latina. Es el vehículo oficial utilizado por el Estado para garantizar educación en la lengua y desde la perspectiva cultural de estos pueblos. Esta modalidad educativa orienta en los modos, las reglas y los mecanismos curriculares y pedagógicos para que los pueblos indígenas y poblaciones afrodescendientes reclamen la educación de sus miembros desde las lenguas y las culturas propias (Corbetta, et al. 2018). 
Para apoyar la educación intercultural bilingüe los Estados utilizan políticas públicas y educativas que permiten la interculturalización de los sistemas sociales y educativos. Al conjunto de políticas públicas y educativas se les define como interculturalidad. El objetivo de la interculturalidad es la creación y ejecución de políticas que hagan efectivo el derecho a la educación de los pueblos mencionados y poner un alto a los sistemas sociales de subalternización y exclusión ciudadana. La interculturalidad educativa busca influenciar a la población blanca-mestiza en reconocer el aporte sociocultural de los pueblos indígenas y poblaciones afrodescendientes, y a su vez el trato de estas como iguales (Corbetta et al., 2018).

A pesar de la brevedad y exactitud que brindan los documentos oficiales para definir estos conceptos, es importante rescatar que a partir de la sociología existen sentidos y usos diversos de estos. Por su abordaje e impacto, se reconocen tres perspectivas distintas: la interculturalidad relacional, funcional o crítica (Walsh, 2008).

La interculturalidad relacional es el contacto o intercambio entre culturas ya sea en condiciones de desigualdad o igualdad. Esta interpretación se preocupa del contacto o relación a nivel individual, pero omite las estructuras sociales, políticas y económicas que protegen las relaciones de superioridad e inferioridad. De este modo, esconde las matrices de colonialidad (Walsh, 2008).

Por otro lado, la interculturalidad funcional reconoce la diversidad y las diferencias culturales con el fin de incluir a los que no son parte de la norma en la estructura sociocultural establecida. Se puede llamar a esta interculturalidad como de tolerancia, de coexistencia, o de diálogo. Esta modalidad de interculturalidad mantiene el orden social establecido y es compatible con la realidad neoliberal presente en los espacios sociopolíticos y socioeconómicos. Esta es una interculturalidad que acepta sin cuestionar (Tubino, 2005). La pasividad característica de la interculturalidad funcional es una poderosa arma de dominación, pues apacigua a los marginados con el fin de controlar el conflicto étnico y así avanzar agendas económicas neoliberales (Walsh, 2008)

Finalmente, la interculturalidad crítica atiende primordialmente a la destrucción de la estructura colonialracial que dio a luz el sistema jerárquico con los blanqueados en la cúspide, y las personas indígenas y afrodescendientes a sus pies. Aquí, la interculturalidad se convierte en el pico y la pala de un proyecto-proceso desde la gente que demanda una sociedad distinta a la subalternidad (Walsh, 2008). La interculturalidad crítica se vale de la pedagogía de-colonial que cuenta con proyectos y procesos epistemológicos, éticos, sociales, políticos y pedagógicos para la legitimación, equidad y respeto de otros modos de pensar y ser. Siendo otros los que vienen de la de-colonialidad (Walsh, 2008). A partir del espíritu de-colonizador se pretende una educación intercultural bilingüe.

\subsection{Contextualización de la educación intercultural bilingüe y la interculturalidad.}

La idea de la educación intercultural bilingüe se desarrolla en América Latina a partir de los años 90 por la necesidad de establecer marcos nacionales de educación bilingüe para el mantenimiento y el rescate de lenguas y culturas indígenas. Bajo el nombre de educación intercultural bilingüe se empezaron a entender dos ideas. Primero, un llamado político indígena para desmantelar la exclusión y fomentar la educación lingüística y cultural desde el indígena para el indígena. Segundo, como el proceso de interculturalidad socio estatal burocratizado legitimista de los derechos étnicos colectivos por medio de mecanismos de regulación educativa para indígenas. Vale rescatar que esta legitimización no fomenta lo propiamente indígena en el sentido sociopolítico, socioeducativo y fundamentalmente cultural (Walsh, 2009).

La intencionalidad de la educación intercultural bilingüe vive implícitamente en el marco internacional de derecho a la educación, pero en algunos casos aboga explícitamente por una interculturalidad relacional o funcional mientras que en otros deja entre dicho que una interculturalidad crítica se puede llevar a cabo. El Comité de los Derechos del Niño, en el Comentario General 1, establece que el propósito de la educación es proteger la dignidad humana innata de toda persona y sus derechos inalienables. Por medio de la educación 
se busca el desarrollo holístico del niño y la niña hasta el máximo de sus capacidades, se potencia su identidad y pertenencia. Además, se les inculca el respeto a los derechos humanos, se promueve su integración a la sociedad y se les orienta hacia el resguardo del medio ambiente (UNICEF, 2006, pp. 9-10). El Comentario 1 delinea el resultado deseado de la educación intercultural bilingüe, pero asume que el Estado es el responsable de ofrecer esta educación que protege y dignifica. El problema, sin embargo, es que el Estado mismo no ha logrado este tipo de educación para con los otros y por ende la lucha por los derechos colectivos de los pueblos marginados.

Previo a la definición del propósito de la educación, en 1966, la Organización de la Naciones Unidas identifica los principios fundamentales del derecho a la educación en el Pacto Internacional de Derechos Económicos, Sociales y Culturales (Organización de las Naciones Unidas [ONU], 1966). Relevante a la educación intercultural bilingüe, se rescata que esta no debe ser discriminatoria, y debe promover la comprensión, la tolerancia, y la paz entre naciones y grupos étnicos/religiosos (ONU, 1966, p. 47). Con la misma visión, la Convención sobre los Derechos del Niño en sus principios rectores identifica la importancia de la igualdad en la forma de no discriminación fundamentada en raza, color, idioma y cultura (Morlachetti, 2015, p. 30). La contribución de la Organización de las Naciones Unidas apunta a una interculturalidad de inclusión y tolerancia que existe dentro de las dinámicas de poder históricamente establecidas. Dicho de otra forma, es una interculturalidad funcional, pues no riñe con las estructuras de opresión y no busca un desmantelamiento del sistema opresor.

La Organización de las Naciones Unidas indica que para lograr el pleno ejercicio al derecho la educación esta debe caracterizarse por su disponibilidad (cantidad suficiente de instituciones y programas de enseñanza), accesibilidad (no discriminación, accesibilidad material, accesibilidad económica), aceptabilidad (los programas de estudio y métodos pedagógicos han de ser aceptables), y adaptabilidad (la educación debe adaptarse a las necesidades sociales y culturales del estudiantado) (ONU, 1999, pp. 2-3).

La adaptabilidad es la premisa que fundamenta la educación intercultural bilingüe, pues tiene como fin el afrontar las necesidades de estudiantes en contextos sociales y culturales variados (ONU, 1999, pp. 2-3). La educación intercultural bilingüe propone encarar la socialización cultural e institucional por medio de la protección a la dignidad humana innata de la niñez, empleando marcos pedagógicos de valoración y respeto cultural.

A partir de la antropología se puede esbozar una aproximación a la pregunta de para qué afrontar las necesidades de estudiantes en contextos sociales y culturales variados. Nacemos y nos criamos en áreas geográficas en la que somos iguales o diferentes a otras personas. Estas diferencias varían en género, etnia, color de piel, idioma, edad, religión, orientación sexual y/o clase socioeconómica. Cada una de las características nombradas forma parte de una identidad social. Así bien, cada una de estas conlleva un valor, ya sea como norma aceptada por la sociedad o como característica no deseada, subordinada o marginada (Harro, 2000).

Harro (2000) propone un marco conceptual para explicar la dinámica de poder entre miembros dominantes y miembros subordinados. Dentro de este marco conceptual establece la institucionalización educativa como un vehículo para reforzar, sancionar, marginalizar, premiar, privilegiar y discriminar a los individuos de la sociedad. Así, las creencias y valores establecidos en la institucionalización de la educación se convierten en credo por medio de la socialización cultural e institucional. Además, esta socialización está plagada de estereotipos y mensajes que han moldeado el cómo y el qué pensamos sobre nosotros mismos y los demás. El objetivo de la socialización cultural e institucional es la justificación de la discriminación y el prejuicio (Harro, 2000).

El ciclo de socialización en América Latina está fundado en una construcción racial que empezó en el colonialismo. El colonialismo es la dependencia política y económica en la cual la soberanía de un pueblo le pertenece a otra nación. A pesar de que estos pueblos colonizados han logrado su soberanía política, en ellos existen, todavía, patrones de poder establecidos en el colonialismo. A esta dinámica de poder racializada se le conoce como colonialidad. En América Latina la colonialidad ha sobrevivido al colonialismo (Maldonado, 
2007). La construcción sociopolítica de América Latina emplea la colonialidad de poder como el esquema mental necesario para crear y mantener patrones de poder mundial de subalternización con fines capitalistas. La colonialidad de poder es la jerarquía en estructuración político-social y económica de las razas en la cual los blanqueados y los mestizos tienen una superioridad natural sobre los indios y los negros (Quijano, 2000).

La dinámica de poder racializada abre paso a la creación de preceptos legislativos. Los pueblos indígenas del Nuevo Mundo logran un marco legal de reconocimiento ancestral cultural como otros basado en su identidad colectiva e idioma. Desde una perspectiva histórica, el Fraile Bartolomé de las Casas, en el año 1542, emprende una campaña para acabar con los abusos de los colonos españoles hacia los indios y reclama que se ponga un alto al exterminio de los indígenas, argumentando que estos tienen en su haber una cultura comparable a cualquier otra del Viejo Mundo y por ende pide su liberación del sistema opresor del colonialismo. Ahora bien, el mismo autor ofrece una solución al problema económico fiscal consecuente de la liberación de los indígenas cuando propone la importación de negros por su predisposición para la esclavitud dada su falta de cultura e inhumanidad (De las Casas, 2019). De las Casas se conoce aún hoy en día como un Procurador de Indios, pero poco se le reclama por haber pagado su libertad con la esclavitud de otros.

Este espíritu de desigualdad entre los indígenas y los negros es una constante desde el colonialismo hasta la modernidad. La Convención Internacional de Labor de 1957 produce la primera póliza internacional (ratificada en 28 países latinoamericanos y asiáticos) con peso legal para el reconocimiento de pueblos indígenas como recipientes de derechos colectivos adicionales por su condición de Primeras Naciones. Consecuentemente, a finales de la década de 1980 las mismas poblaciones indígenas beneficiadas por la póliza demandan su revisión, lo que inicia la reforma legal en los 1990 (Walsh, 2014). La revisión provee una oportunidad para la inclusión de las poblaciones afrodescendientes. Tres ejemplos de inclusión de afrodescendientes en el marco legal emergen en América Latina: Colombia, 1991; Ecuador, 1998; y Bolivia, 2008. Estos tres países añaden estatutos a las leyes de derechos colectivos indígenas para incluir derechos colectivos de negros, mulatos y afrodescendientes.

Previo a estos tres ejemplos, las poblaciones afrodescendientes estaban invisibilizadas en los marcos legales en América Latina. Una de las teorías que explica esta invisibilidad sugiere que las poblaciones indígenas han sido percibidas como grupos etno-culturales, mientras que las poblaciones negras han sido clasificadas como racialmente distintas (Greene, 2007). De este modo, las poblaciones negras que han indigenizado sus reclamos han logrado avanzar y encontrar lugar para sus demandas (Restrepo, 2002).

La adaptabilidad educativa atiende las necesidades de estudiantes en contextos sociales y culturales variados por medio de prácticas pedagógicas. Desde una perspectiva etnográfica y antropológica, Dietz (2012) clasifica las prácticas pedagógicas empleadas en servicio de la interculturalidad y la diversidad en la educación respecto al ¿para qué se enseña? 1 (2012) se refiere a cada modalidad organizada de acuerdo a sus objetivos, en los siguientes términos:

- Asimilar. Ejemplos clásicos de este tipo de educación se encuentran en los procesos de americanización en los Estados Unidos de América antes de los movimientos multiculturales de los años 1970. Esta modalidad busca adaptación cultural del estudiantado hacia un monoculturalismo presente en la estructura dominante. Otro ejemplo de este modelo educativo es el movimiento del nacionalismo francés, o en la postura ideológica española que niega la importancia de la especificidad cultural en las escuelas.

- Compensar: La pedagogía para extranjeros en Alemania tiene por objetivo la superación de desventajas lingüísticas o culturales. Su objetivo es propiciar la homogeneidad en el estudiantado.

- Diferenciar o biculturalizar: Los sistemas educativos holandés y belga introducen el concepto de enseñanza en la lengua y cultura de origen desde la perspectiva de que todas las comunidades con características lingüísticas y étnicas diferentes a la norma nacional tienen derecho a una educación específica. En estos casos se enseña al estudiantado su cultura de origen, que muchas veces acaba 
siendo la cultura del país de sus padres. Otro ejemplo, en América Latina, es la educación bilingüe y bicultural que normaliza hacia la lengua dominante mientras que preserva el idioma dominado.

- Tolerar: La educación para la tolerancia fomenta el diálogo y el respeto entre culturas, desarrollando valores y aptitudes de solidaridad. Esta modalidad educativa incluye al alumnado de la cultura mayoritaria, pero tiene como fin la asimilación del otro.

- Prevenir racismo: La educación antirracista se desarrolló primordialmente en el Reino Unido con el fin de enseñar al estudiantado a percibir estructuras sociales, políticas y estatales fundadas en ideologías racistas.

- Interactuar: La educación para interactuar emplea una pedagogía dialógica y colaborativa en la cual los salones de clase reflejan la diversidad cultural del contexto real del alumnado. Desde esta construcción cultural el estudiantado recibe poder y responsabilidad de su propia educación. Así, el profesorado se convierte en un mediador de aprendizaje complejo.

- Empoderar: La educación que empodera se enfoca en grupos históricamente excluidos por medio de la creación de currículo pertinente. Esta modalidad se conoce también como educación para la acción afirmativa.

- Descolonizar: La educación descolonizadora parte de los conceptos de colonialidad y colonialismo (Maldonado, 2007) discutidos anteriormente. Tiene como fin la destrucción de sistemas de opresión en busca de una educación que pedagógica y didácticamente sea por y para los que han sido marginados. Esta es educación que busca la examinación de la historia, los saberes y las perspectivas para encontrar otras formas de entender y conocer el mundo.

Los Estados acaban con implementaciones educativas que son incoherentes en las cuales se cree que se está haciendo una cosa (des-colonizar) pero en efecto se está haciendo otra (mantener sistemas de opresión). La incoherencia proviene de la incomprensión de la interculturalidad para destruir la colonialidad de poder (Quijano, 2000) y que esto se logra solamente en la medida en la que se empleen prácticas pedagógicas alineadas a este objetivo (Dietz, 2012).

Desde la síntesis de los conceptos educación intercultural bilingüe e interculturalidad, la segunda sección expone un referente histórico y actual de las personas afrocostarricenses a partir de la educación intercultural bilingüe y la interculturalidad en Costa Rica.

\section{LA PERSONA AFROCOSTARRICENSE DESDE LA EDUCACIÓN INTERCULTURAL BILINGÜE Y LA INTERCULTURALIDAD.}

Las personas afrocostarricenses nacen como consecuencia de un préstamo del gobierno inglés al costarricense, para empezar la construcción de un ferrocarril entre el valle central y el atlántico en 1871. Esta obra inicia una corriente laboral migratoria en la provincia de Limón. Los primeros en llegar a Limón para trabajar en el ferrocarril fueron ingleses, portugueses e italianos, pero estos no lograron soportar las condiciones climáticas y ambientales de la zona. De esta forma, el 20 de diciembre de 1872 marcó la llegada del primer grupo de trabajadores isleños del Caribe, principalmente jamaiquinos (Ministerio de Educación Pública de Costa Rica, 2009).

Para el año 1873 en Limón se encuentran más de ochocientos trabajadores provenientes de Jamaica. En 1874 el gobierno costarricense queda sin fondos para continuar la construcción del ferrocarril, como consecuencia muchos de los trabajadores jamaiquinos son despedidos. El Estado, que desea no perder completamente esta mano de obra, ofrece la entrega de lotes de $50 \times 50$ para aquellos jamaiquinos que deseen quedarse en el país. Se origina entonces el primer asentamiento formal de afrodescendientes en Costa Rica y con éste, el establecimiento de normas sociales, culturales, religiosas y educativas arraigadas a sus raíces lingüísticas, raciales y étnicas (Ministerio de Educación Pública de Costa Rica, 2009). 


\subsection{La ausencia de las personas afrocostarricenses en el discurso nacional actual respecto a la educación.}

En Costa Rica, el informe del Estado de la Educación (Consejo Nacional de Rectores [CONARE], 2019), no ofrece un discurso oficial para la educación intercultural bilingüe. El tercer capítulo: educación primaria, y el cuarto capítulo: educación secundaria, no evidencian una pedagogía hacia la diversidad cultural y lingüística nacional. La ausencia de la temática de educación intercultural bilingüe es sorprendente ya que el informe Programa Estado de la Nación (CONARE, 2015) en su quinto capítulo: desigualdades en el rendimiento sistemático educativo costarricense, pone de manifiesto cómo las poblaciones con diversidad cultural (ya sean afrodescendiente, migrante o indígena), tienen sistemáticamente bajo rendimiento académico en comparación a la norma nacional. El informe atribuye el bajo rendimiento al índice de pobreza que experimentan estas poblaciones.

Mi primer interrogante respecto a la falta de un discurso nacional en el informe del 2019 (CONARE, 2019) apuntó a si la decisión de la exclusión se debía a que la población tenía un porcentaje tan bajo que no repercutía en las tendencias generales. Para evacuar la duda, analicé el reporte Costa Rica: Indicadores de Educación y de Contexto (INEC y UNICEF, 2014) que tomó información del censo desarrollado en el año 2011 para identificar las principales características de la población, hogares y viviendas en diferentes áreas geográficas con el fin de diagnosticar la educación nacional y su relevancia social y económica. Este reporte revela que en Costa Rica 7,8 por ciento de la población nacional es afrodescendiente.

El reporte no distingue cuántas personas de cada subcategoría tienen estudios superiores. Aun así, hace un análisis de porcentajes en cada región geográfica, y menciona cuántas personas de la población total de la región geográfica tienen estudios superiores. Tomando en cuenta estos porcentajes, y observando tendencias educativas por área geográficas con concentración de población afrodescendiente se rescatan los siguientes datos (INEC y UNICEF, 2014):

- Dirección Regional de Educación de Cartago, con una población negra de 0,4 por ciento y una población mulata de 5,1 por ciento. En esta zona un 18,1 por ciento de la población geográfica total tiene estudios superiores

- Dirección Regional de Educación de Alajuela, con una población negra de 0,5 por ciento y una población mulata de 5,6 por ciento. En esta zona un 17,9 por ciento de la población geográfica total tiene estudios superiores.

- Dirección Regional de Educación de Heredia, con una población negra de 0,6 por ciento y una población mulata de 6,1 por ciento. En esta zona un 27,8 por ciento de la población geográfica total tiene estudios superiores.

Mi segunda interrogante referente al desempeño académico de las personas afrocostarricenses apuntó a su diversidad lingüística. Las personas afrocostarricenses hablan tres idiomas: el español, el criollo limonense, y el inglés limonense estándar (Zimmer, 2011). Lo cual hace a esta población no solo culturalmente diversa, sino también lingüísticamente diferente a la población general del país. Por tanto, mediante una comunicación electrónica personal al buzón general del Departamento de Educación Intercultural, que ejerce bajo la Dirección de Desarrollo Curricular del Ministerio de Educación Pública de Costa Rica, se hizo una entrevista a actores políticos de la educación intercultural. Ante la pregunta de que, si existe una propuesta curricular universal del MEP diseñada para apoyar el desarrollo del inglés limonense estándar y del español, la respuesta evidenció que no se cuenta con un programa, plan o proyecto institucional que apoye específicamente el desarrollo del inglés criollo limonense (Departamento de Educación Intercultural, comunicación personal, 2019). 
Ahora bien, se mencionó que se lleva a cabo capacitación docente a nivel nacional para sensibilizar, fortalecer y mantener las lenguas que son parte de la cultura afrocostarricense y del país. Se reconoció que, en algunos centros educativos de la provincia de Limón, a nivel comunal, se ejecutan algunos trabajos de revitalización de la lengua. Estos esfuerzos son parte de una colaboración con la Escuela de Literatura y Ciencias del Lenguaje de la Universidad Nacional (Departamento de Educación Intercultural, comunicación personal, 6 de septiembre, 2019).

$\mathrm{Ni}$ el porcentaje respecto a la población total, ni la diversidad lingüística me permitieron entender la exclusión en el discurso nacional actual. En contraste, los archivos históricos constatan que entre 1880 y 1915 en Limón se establecieron las escuelas formales de inglés. En estas instituciones parroquiales; la Primer Iglesia Bautista, la Iglesia Metodista, la Episcopal y la Adventista, se enseñaba en la lengua materna del estudiantado en las áreas curriculares de matemáticas, ciencias, literatura universal, literatura inglesa, geometría, álgebra, historia universal, poesía, teatro, dibujo, gramática, y ortografía. El apoyo curricular se daba en la utilización de libros de texto ingleses. Las escuelas formales de inglés educaron a niños afrodescendientes desde 1880 hasta la década de 1950. Después de 9 años de instrucción formal del estudiantado se graduaban con un caudal de conocimiento comparable a un bachiller en la actualidad (Ministerio de Educación Pública de Costa Rica, 2009).

La revolución de 1948 da a luz a un movimiento nacionalista que afecta al Caribe costarricense en cuanto a que la revolución declara la educación como una prioridad nacional. En Limón esto significó el establecimiento de escuelas públicas oficiales y obligatorias. Estas escuelas públicas obligatorias son en español.

Los educadores enviados desde el valle central no estaban preparados para entender cultural o lingüísticamente a sus pupilos, y las personas afrocostarricenses se ven marginadas en políticas que los obligaban a no utilizar el inglés como medio de comunicación en las escuelas. La prohibición del uso del inglés impacta negativamente la identidad del afrocostarricense en que desvaloriza la lengua y la cultura materna y a su vez introduce al educador y al Estado como figuras de autoridad (Pizarro, 2003).

La declaración de la educación como prioridad nacional a consecuencia de la revolución de 1948 beneficia al blanco y al mestizo costarricense, pues por primera vez hace de la educación un bien disponible; pero obliga a las personas afrocostarricenses a igualarse hacia abajo ya que la educación pública y obligatoria costarricense fue discriminatoria cultural, étnica y lingüísticamente, empleó métodos pedagógicos inaceptables y no se adaptó a las necesidades sociales, culturales y sociales del estudiantado.

El propósito de la educación es "potenciar la identidad y pertenencia de los estudiantes" (UNICEF, 2006, pp. 9-10). Por esta razón, la educación pública monolingüe ha impactado negativamente a las personas afrocostarricenses. El lenguaje es en sí mismo una expresión de cultura y creatividad humana, por medio del lenguaje se dan a conocer los saberes, creencias e ideas con una perspectiva etnográfica. El lenguaje es también el vehículo para experimentar el contexto en el que la persona se desarrolla, siendo estos los saberes extralingüísticos o conocimientos del mundo transversal en la expresión lingüística. El lenguaje es también un fenómeno social, utilizado por los miembros de una comunidad para comunicarse, aprender, evaluar y analizar su realidad pasada, presente y futura. La lengua y la cultura están en el centro de los fenómenos de identidad (Gomes y Catchcart, 2011). El lenguaje es el medio utilizado para preservar la cultura y las tradiciones; por este motivo, la pérdida de lenguaje también significa pérdida de cultura e identidad (Baker, 2001).

Existe una asociación indiscutible entre lenguaje e identidad, porque el lenguaje es el medio utilizado por el individuo para entender quién es y su contexto (Pierce, 1995; Norton, 2000). La construcción de identidad es un proceso sociocultural engranado en prácticas discursivas. De esta forma, los hablantes de múltiples lenguajes o aprendices de un idioma dominante experimentan tensión entre el discurso dominante social y el discurso marginado socialmente. El lenguaje, visto de esta forma, es el centro cultural de la construcción de identidad (Bucholtz y Hall, 2004). 
En esta línea, Singleton (2015) expone que la raza o etnia del estudiantado en instituciones públicas y los valores asignados a estas razas o etnias impactan la capacidad de estas personas para acceder a la educación a la que tienen derecho. En su trabajo, Courageous Conversations about Race, Singleton (2015) propone un protocolo para institucionalizar y socializar a nivel cultural por medio de conversaciones acerca del impacto de raza o etnia. Parte del protocolo promueve la creación de biografías raciales en las que el estudiantado, el profesorado, la administración y las personas encargadas de la legislación educativa, escriben autobiografías utilizando una perspectiva racial.

El objetivo del autor es instar el diálogo honesto y abierto acerca de qué influye en que el estudiantado no tenga un buen rendimiento académico sea esto lo que la sociedad ha institucionalizado (colonialidad de poder que es una jerarquía de poder racializada), o por quienes son (personas en los peldaños más bajos de la jerarquía). La propuesta de re-institucionalización educativa de este autor, ha sido fuertemente criticada en la base que requiere que los agentes de grupos dominantes acepten responsabilidad social de lo que el texto identifica como el privilegio de ser blanco (Singleton, 2015). El autor, desde sus aportes ofrece puntos de entrada en busca de pedagogías críticas para el enaltecimiento del estudiantado negro y afrodescendiente.

Entender la trayectoria de la educación intercultural bilingüe en servicio de los afrocostarricenses requiere de un análisis fundado también en la antropología. En la temática de procesos étnicos, Bonfil (1991) hace un importante aporte con la Teoría de Control Cultural, el investigador sugiere que existen elementos culturales que son propios y otros que son ajenos. De igual forma cada grupo étnico toma decisiones propias o ajenas. De este modo, los grupos étnicos que toman decisiones propias acerca de elementos culturales propios son parte de culturas autónomas. Los grupos étnicos que toman decisiones propias acerca de elementos culturales ajenos se identifican como culturas apropiadas. Los grupos étnicos para con quienes otros o ajenos toman decisiones acerca de sus propios elementos culturales se denominan culturas enajenadas. Finalmente, los grupos étnicos que están subyugados a que ajenos tomen decisiones respecto a los elementos culturales de terceros se subscriben a culturas impuestas. Desde la Teoría de Control Cultura, y contextualizado en el Ciclo de Socialización de Harro (2000) aunado con la colonialidad de poder (Quijano, 2000), se argumenta que los afrocostarricenses -en ausencia de control político centralizado nacional- ejercieron como un grupo étnico autónomo en el área de educación ya que tenían control y poder de decisión respecto a los contenidos y los modos de educar a sus hijos. Una vez que la educación pública se convirtió en obligatoria el aspecto educativo los situó como grupo étnico enajenado.

En la actualidad, Costa Rica es reconocida globalmente como una nación que valora la educación y el alfabetismo. Ahora bien, se nota rezago al analizar el porcentaje de alfabetismo en forma desagregada por grupo étnico racial, y diferenciando entre población urbana y rural. Si bien es cierto que la población total costarricense tiene una tasa de alfabetismo de $96.8 \%$, la población urbana goza una tasa de $97.9 \%$ y la rural de 93.7\%; la población afrodescendiente total tiene una tasa total de alfabetismos de $96.1 \%$, la población urbana del mismo grupo apunta a una tasa de $97.4 \%$ pero la rural documenta solamente $92.4 \%$ (Programa de las Naciones Unidas para el Desarrollo [PNUD], 2013a).

Con una trayectoria similar, el análisis de promedio de años de escolaridad de la población de 25 años demuestra diferencias entre la población total y los datos desagregados por grupo étnico racial, y en vista a población urbana o rural. En este caso, la población total costarricense tiene un promedio de 8.5 años de escolaridad, en las zonas urbanas el promedio es de 9.2 años y en la rural 6.4 años. Para los afrodescendientes se apunta a una disparidad en la cual la población total promedia 7.7 años de escolaridad. En la zona urbana se acusan 8.3 años promedio de escolaridad y en la rural solamente 5.9 (PNUD, 2013a).

Para solventar este rezago el gobierno costarricense en el documento Visión 2030 (PNUD, 2017) propone: (1) la creación de un currículo que enaltezca la identidad afrodescendiente, (2) la recuperación del inglés criollo limonense, y (3) la garantía de acceso a educación de calidad, y declaración de la Provincia de Limón como bilingüe. Aun así, no existe evidencia de un plan concreto para el logro de estos objetivos. Además, en la entrevista con personas funcionarias del Departamento de Educación Intercultural del Ministerio de 
Educación Pública se confirma que no existe un plan nacional para cumplir estas metas (Departamento de Educación Intercultural, comunicación personal, 6 de septiembre, 2019). Una revisión cuidadosa de los recursos disponibles en la página electrónica del MEP resultó en que no existe un solo recurso curricular o didáctico disponible respecto al bilingüismo para las personas afrocostarricenses o educación intercultural para el mismo grupo (Ministerio de Educación Pública de Costa Rica, 2018).

Las personas afrocostarricenses han sido parte de una encrucijada educativa dado que la educación pública costarricense en Limón ignora y desprecia las tradiciones culturales africanas e inglesas (Duncan y Meléndez, 2011, pp. 111, 120-121). El ciclo de socialización (Harro, 2000) no solamente afectó a las personas afrocostarricense académicamente, pero también inició un sistema de percepciones y actitudes de la población general hacia las personas afrocostarricenses.

\subsection{Percepción y actitudes hacia la población afrodescendiente}

Las personas afrodescendientes logran el estatus de ciudadano en Costa Rica hasta finales de la década de 1940 (Ministerio de Educación Pública de Costa Rica, 2009), pero la ciudadanía no logra la igualdad social. En 1939, viendo la posibilidad de la ciudadanía de las personas afrodescendientes, el científico costarricense Clodomiro Picado sentenció en el Diario de Costa Rica:

¡NUESTRA SANGRE SE ENNEGRECE!, y de seguir así, del crisol no saldrá un grano de oro sino un pedazo de carbón. Puede que aún sea tiempo de rescatar nuestro patrimonio sanguíneo europeo que es lo que posiblemente nos ha salvado hasta ahora de caer en sistemas de africana catadura, ya sea en lo político o, ya en aficiones que remedan el arte o la distinción, en tristes formas ridículas Quizás Ud. cuya voz prestigiada es oída por los humanistas de valer que aún quedan en estas regiones, logre ayudar a señalar el precipicio hacia el cual nos encaminamos. (Molina, 2015, p. 131).

Poco ha cambiado en setenta y tres años. En febrero del 2012, el artículo Racismo y matonismo en la escuela enunció:

\footnotetext{
Una niña negra de 13 años se bañó en talco para disimular el bello color de su piel. Sus condiscípulos, empeñados en hacerle la vida imposible, la acosan con comentarios racistas al punto de infundirle rechazo a la escuela, episodios de ira en la casa y contribuir a la pérdida de dos años reprobados. La situación ofende el más básico sentido de la justicia y alerta sobre dos problemas innegables: la existencia de racismo en nuestra sociedad y la aparición, cada vez más frecuente, del matonismo en los centros educativos. (Racismo y matonismo en la escuela [Editorial], 2012, párr. 1).
}

El artículo El negro en la Costa Rica de Hoy, (Duncan, 2005) documenta, a partir de la sociología, una gran serie de micro y macro agresiones hacia las personas afrocostarricenses. Todas estas agresiones racistas, despectivas y de suma desconfianza. Se nota por ejemplo el "criterio de que todos los negros son borrachos, parranderos e incapaces de trabajo intelectual” (Duncan, 2005, p. 402). Se documentan además instancias en las que se le ha negado a un afrocostarricense la compra de una propiedad por miedo a la desvalorización de otras propiedades contiguas, o la negación de matrícula a una niña negra en una escuela privada por miedo al "mal negocio" (Duncan, 2005, p. 402).

Duncan (2005) explica que el blanco-mestizo utiliza la estrategia de negar la existencia de sus propios prejuicios para con afrocostarricense, negando así la propia existencia de este. Advierte, además, del peligro de esta estrategia dado que en la negación se obstruye el camino a diálogo (Duncan, 2005). Esta negación es también parte de la interculturalidad relacional en la que en cuanto se acusa a una persona de prácticas opresoras esta puede refugiarse en expresiones como: ;Cómo se le ocurre que yo soy racista! Tengo un amigo negro.

Fuera de los casos extremos que se publican en periódico y revistas, en el 2010, el Instituto de Estudios Sociales en Población (Idespo) y la UNICEF desarrollaron una encuesta sobre Percepciones y Actitudes de la Población Costarricense hacia la Población Afrodescendiente. El análisis estadístico de la encuesta explica la persistencia histórica de prejuicios sociales en contra de las personas afrocostarricenses y sus manifestaciones 
(Corredor Cultural del Caribe, 2013). A modo de ejemplo, entre los prejuicios documentados 79\% de los entrevistados reportaron que las personas negras o afrodescendientes se caracterizan por ser personas alegres a las que les gusta mucho el carnaval y la fiesta (Instituto de Estudios Sociales en Población, 2009).

El tema de percepción es importante y ha sido estudiado. El Informe Nacional sobre Desarrollo Humano, Aprendiendo a vivir juntos: Convivencia y desarrollo humano en Costa Rica (PNUD, 2013b) exploró actitudes de la población general costarricense respecto a la población afrocostarricense. El informe reportó que entre los entrevistados existía una tendencia de rechazo a las prácticas excluyentes. Solamente $10 \%$ de los entrevistaron estuvieron muy de acuerdo y de acuerdo con que "la única manera de respetar las diferencias entre los afrocostarricenses y el resto de los costarricenses es que cada grupo viva aparte" (PNUD, 2013b, p. 195). Pero al mismo tiempo, $37 \%$ de los entrevistados reportaron estar muy de acuerdo y de acuerdo con que "si los afrocostarricenses quieren ser aceptados entonces deben de adaptarse más a las tradiciones y costumbres de la mayoría de los costarricenses" (PNUD, 2013b, p. 195). Otra incoherencia se demuestra en que $42 \%$ de los entrevistados estuvieron muy de acuerdo y de acuerdo con el enunciado "para que puedan adaptarse mejor los afrocostarricenses deberían de intentar hablar más español y menos en su idioma” (PNUD, 2013b, p. 195), pero $67 \%$ de los entrevistados asintieron que "nuestro sistema educativo debería promover que los afrocostarricenses mantengan su propia cultural” (PNUD, 2013b, p. 195). Es incomprensible que un país como Costa Rica que goza de la reputación de ser una nación pacifista y amante de la educación, uno de los países más felices del mundo, se caracteriza por tendencias racistas (Corredor Cultural del Caribe, 2013).

$\mathrm{La}$ ambivalencia de las respuestas de los entrevistados ejemplifica la incongruencia entre lo que se cree de uno mismo, en este caso que se rechazan las prácticas excluyentes; pero que se rechazan solamente si se homogeniza a la persona afrocostarricense para que sea más cercana en sus modos o a la norma nacional blanco-mestiza.

\subsection{La presencia de las personas afrocostarricenses en el discurso nacional político. Interculturalidad.}

La interculturalidad es el conjunto de políticas públicas y educativas que promueven cambio en los sistemas sociales y educativos. La interculturalidad busca la reforma social haciendo efectivo el derecho a la educación de los pueblos históricamente marginados por etnia y lengua. La interculturalidad busca el desmantelamiento de la subalternización y exclusión ciudadana (Corbetta et al., 2018).

En Costa Rica, el primer proyecto de ley que apunta hacia la antidiscriminación se publicó en 1960, y cuenta con 17 políticas públicas y educativas, esto de acuerdo con el Plan Nacional para Afrodescendientes 2015-2018 (Comisionado Presidencial para Asuntos de la Afrodescendencia, 2015):

1. 1960. Ley número 2694. Ley antidiscriminación. Esa ley objeta la discriminación racial y promueva igualdad de acceso en empleo u ocupación

2. 1968. La ley número 4230. Ley contra la discriminación. Esta ley decreta multas para quienes prohibieran acceso a educación, asociaciones, centros de diversión o lugares públicos a personas por motivos raciales o étnicos.

3. 1970. La ley número 4573, Código Penal de Costa Rica de 1970. Esta ley sanciona multas a quienes sean parte de prácticas discriminatorias raciales.

4. 1973. La ley número 5360. Esta ley elimina restricciones a la migración con bases en características raciales o étnicas.

5. 1980. Decreto Ejecutivo número 11938-E. Declaración del Día del Negro, 31 de agosto de cada año.

6. 1994. Ley número 7426. Esta ley sustituye Día de la Raza convirtiéndolo en el Día de las Culturas con el fin de enaltecer el carácter pluricultural y multiétnico del pueblo costarricense, e incluye en el 
artículo 3, que en los programas de estudios del primer y segundo ciclos, se deberán incluir todos los componentes culturales y étnicos acordes con el carácter pluricultural y multiétnico del pueblo costarricense.

7. 1997. Ley número 7711. Ley de eliminación de la discriminación racial en los programas educativos y en los medios de comunicación. De suma importancia para la educación costarricense ya que ordena al Consejo Superior de Educación la revisión de textos escolares para eliminar formas de discriminación y además incorporar elementos culturales e históricos afrodescendientes. Esta ley hace ilegal la comunicación escrita, radial y televisiva con tendencias racistas.

8. 2000. Ley 8054. Ley de la diversidad étnica y lingüística. De esta ley surge el Día Nacional de la Diversidad Étnica y Lingüística, con el fin de nombrar las contribuciones de poblaciones históricamente marginalizadas por etnia y lengua.

9. 2001. Ley número 8107. Como parte del Código de Trabajo, esta ley prohíbe la discriminación por edad, género, etnia o religión.

10. 2005. Decreto número 32338-MEP. Establecimiento de la Comisión Nacional de Estudios Afro Costarricenses. Esta comisión tiene por objetivos la integración de cosmovisión, filosofía y pensamiento de los afrodescendientes en el proceso nacional educativo.

11. 2011. Ley número 8938. Declaración del 31 de agosto como Día de la Persona Negra y la Cultura Afrocostarricense.

12. 2011. Decreto ejecutivo 36776-RE. Creación de la Comisión Interinstitucional para el Seguimiento e Implementación de las Obligaciones Internacionales de Derechos Humanos (CCIIDDHH), para promulgar la Política Nacional para una Sociedad Libre de Racismo.

13. 2014 Decreto número 38114. Aprobación de la Política Nacional para una Sociedad Libre de Racismo y su Plan de Acción.

14. 2014. Decreto legislativo número 9223. Reconocimiento de los Derechos de los Habitantes del Caribe Sur.

15. 2015 Decreto número 38629-MCJ-MEP. Declaración del asiento histórico de los afrocostarricenses como la Puebla de los Pardos

16. 2015. Decreto 388-35-RE de la Presidencia de la República. Nombramiento del Comisionado Presidencial para Asuntos de la Afrodescendencia.

17. 2015. Directriz número 022-P. Esta dicta que los Ministerios, dependencias del Gobierno Central e Instituciones Descentralizadas han de formular políticas públicas de acuerdo con el Programa de actividades del Decenio Internacional para los Afrodescendientes. (Comisionado Presidencial para Asuntos de la Afrodescendencia, 2015).

Aún con esta infraestructura legal que debiera asegurar el acceso pleno de la población afrodescendiente en todas las actividades económicas, sociales y políticas, el reporte Situación socioeconómica de la población afrodescendiente de Costa Rica (PNUD, 2013a) analiza la tipografía de los hogares para encontrar que 89.4\% de la población total vive en albergues dignos sin carencia, pero solo $84.9 \%$ de las personas afrodescendientes viven bajo iguales condiciones. Así mismo, $90 \%$ de la población total tiene acceso a viviendas con estructuras físicas sanitarias sin carencia, en comparación a 87.2\% de la población afrodescendiente en la misma categoría. El reporte rescata que en Costa Rica 5.9\% de la población total vive en pobreza extrema, pero si se desagregan los datos $8.5 \%$ de los afrodescendientes pertenecen a este grupo.

El informe Visión 2030 Poblaciones excluidas en Costa Rica (PNUD, 2017) expone que dentro del Programa de Acción del Decenio Internacional para la Población afrodescendiente es preciso poner fin a la pobreza promoviendo el crecimiento económico, sostenible y empleo pleno para todas las personas (objetivos 1,7 y 8 ). El mismo documento apunta a la necesidad de crear medidas especiales para asegurar igualdad de oportunidades y participación de todos los ciudadanos. 
El Decenio Internacional para los afrodescendiente desde la temática de reconocimiento destaca el desafío que presenta el derecho a la educación. Para las personas afrocostarricenses el acceso, la pertenencia y el egreso de educación está vinculado con condiciones generales de vida, y por ende tiene índices más bajos que la población en general. Para contrarrestar este desafío, el gobierno costarricense propone cuatro objetivos de prioridad relacionados a la educación del afrocostarricense: (1) Creación de un currículo pertinente que enaltece la identidad afrodescendiente, (2) Recuperación del inglés criollo limonense utilizando de instituciones pedagógicas para ello. (3) Garantización el acceso a educación de calidad, y declaración de la Provincia de Limón como bilingüe. (4) Ofrecimiento de educación técnica que forme en habilidades de empleabilidad (INA, MEP, privada) (PNUD, 2017).

A pesar de la importancia de los objetivos de prioridad (PNUD, 2017), en la entrevista con personas funcionarias del Departamento de Educación Intercultural del Ministerio de Educación Pública Costarricense se mencionaron solamente proyectos de ley y no planes específicos a corto o largo plazo. Se mencionó la Ley 9619 que faculta al Ministerio de Educación Pública y al Ministerio de Cultura y Juventud a promover actividades que contribuyan al reconocimiento y la importancia del inglés criollo limonense. Esta misma ley declara el 30 de agosto de cada año como día para la celebración de la Lengua Criolla Limonense (Departamento de Educación Intercultural, comunicación personal, 6 de septiembre, 2019).

La misma entrevista apuntó que tanto la Política educativa (La persona: centro del proceso educativo y sujeto transformador de la sociedad) como la Política Curricular (Fundamentación Pedagógica de la Transformación Curricular) vigentes, son políticas integrales donde se insiste en la facultad y necesidad de que el profesorado de cualquier parte del país contextualice el currículo de acuerdo a su realidad más cercana o al contexto sociocultural donde se desempeña (Departamento de Educación Intercultural, comunicación personal, 6 de septiembre, 2019). Finalmente, el decreto ejecutivo №35513, en el Artículo 16 dicta que:

Las Direcciones Regionales de Educación, de conformidad con la política educativa y los lineamientos establecidos para tales efectos, serán responsables de promover la educación intercultural como instrumento para la contextualización de la política educativa, así como para enaltecer y fortalecer el carácter pluricultural y multiétnico de nuestra sociedad (Departamento de Educación Intercultural, comunicación personal, 6 de septiembre, 2019).

Desgraciadamente, no se encontró evidencia de los planes actuales para la materialización de los objetivos referenciados en los planes de ley. Tener un día para la celebración de la Lengua Criolla Limonense, no desarrolla destrezas lingüísticas en dos idiomas. Insistirle al profesorado que contextualice el currículo no resulta en educación contextualizada, especialmente cuando una revisión de los planes educativos de las universidades públicas costarricenses en las carreras de educación demostró una falta de intencionalidad respecto a la enseñanza explícita de la población afrodescendiente. Para nombrar algunos otros ejemplos, la investigadora no encontró el reporte ordenado al Consejo Superior de Educación en la ley $\mathrm{N}^{\circ} .7711$ en la página electrónica del Consejo Superior de Educación de Costa Rica (2019) ni en la página electrónica del Ministerio de Educación Pública de Costa Rica (2019). El objetivo de la ley fue la revisión de textos escolares para eliminar formas de discriminación y además incorporar elementos culturales e históricos afrodescendientes. De igual forma, aunque la ley $\mathrm{N}^{\circ} .8054$ promueve la diversidad étnica y lingüística esta no se ha resultado en educación bilingüe para las personas afrocostarricenses.

\section{ApUnTES FinALES}

La desigualdad existe en Costa Rica. Evidencia de esto es la predictibilidad racializada en el rendimiento académico, acceso económico y percepción social. Este ensayo reconoce que la educación es un catalizador de la sociedad y por ende justifica y propone la implementación de interculturalidad y de programas interculturales bilingües como instrumentos de desarrollo del individuo por medio de pedagogías, modos y saberes desde y para sí. El panorama actual costarricense, a pesar de sus múltiples planes de gobierno, 
reproduce patrones de exclusión académica, económica, sociocultural y además condiciona la persistencia de prejuicios hacia las personas afrocostarricenses. Abogo por espacios que nos permitan autonomía para tomar decisiones propias de elementos culturales propios, siendo los más importantes la educación de nuestros hijos y el desarrollo de nuestros idiomas e identidades. La pedagogía crítica, dialógica, des-colonizadora requiere de aptitudes e ideologías que aún no son parte del colectivo social costarricenses. Por este motivo, el primer paso es reconocer esta carencia.

Es esencial que la acción educativa intercultural bilingüe en Costa Rica sea reorientada. Costa Rica no puede, y no debe, aceptar la sistematización de la pérdida del espíritu social que ocurre cada vez que un niño afrocostarricense se nota ausente en el texto, silenciado en la rima, y opacado en la retórica.

Institucionalmente, en vista a la oferta legal, los reportes y los planes actuales; se puede decir que Costa Rica está aún lejos de cumplir el objetivo de la interculturalidad. En esta elaboración existen oportunidades únicas para el crecimiento del costarricense como colectivo social. Cierro instando a la persona lectora a suspender una realidad nacional de disonancia entre lo que dice el papel y lo que se vive, para recrear una realidad alternativa en igualdad. Cuando la educación y la vivencia diaria de todos los ciudadanos sea capaz del desarrollo del individuo y de la valoración cultural del yo, el valor agregado de la conciencia nacional crecerá exponencialmente.

\section{REFERENCIAS}

Baker, C. (2001). Foundations of Bilingual Education and Bilingualism. Clevedon: Multilingual Matters.

Bonfil, G. (1991). La teoría del control cultural en el estudio de procesos étnicos. Estudios sobre las culturas contemporáneas, 4(12), 165-204.

Bolseguí, M. y Fuguet, A. (2006). Construcción de un modelo conceptual a través de la investigación cualitativa Sapiens. Revista Universitaria de Investigación, 7(1), 207-229. Recuperado de http://www.redalyc.org/articulo .oa?id=41070114

Bucholtz, M. y Hall, K. (2004). Language and Identity. En A. Duranti (Ed.). A Companion to Linguistic Anthropology (pp. 369-394). California: Blackwell Publishing.

Cisterna, F. (2005). Categorización y triangulación como proceso de validación del conocimiento en investigación cualitativa. Revista Theoria, 14(1), 61-71.

Comisionado Presidencial para Asuntos de la Afrodescendencia. (2015). Plan Nacional para Afrodescendientes 2015-2018. San José: Presidencia de la República de Costa Rica.

Consejo Nacional de Rectores. (2015). Informe Estado de la Nación. San José: Consejo Nacional de Rectores. Recuperado de http://www.estadonacion.or.cr/educacion2015/

Consejo Nacional de Rectores. (2019). Informe Estado de la Educación. San José: Consejo Nacional de Rectores.

Consejo Superior de Educación de Costa Rica. (2019). El Consejo Superior de Educación. Recuperado de http://cse .go.cr/

Corbetta, S., Bonetti, C., Bustamante, F. y Vergara, A. (2018). Educación intercultural bilingüe y enfoque de interculturalidad en los sistemas educativos latinoamericanos. Santiago: Organización de las Naciones Unidas.

Corredor Cultural del Caribe. (2013). Afrodescendencia en Costa Rica: Sociedad Multiétnica y Pluricultura. Recuperado de https://bit.ly/34W5kdO

De Las Casas, B. (2019). Brevisima relación de la destrucción de las Indias. Puerto Rico: Textos Clásicos Universales.

Dietz, G. (2012). Multiculturalismo, Interculturalidad y Diversidad en Educación. Una aproximación antropológica. México: Fondo de Cultura Económica.

Departamento de Educación Intercultural. (6 de septiembre, 2019). Entrevista con personas funcionarias del Departamento de Educación Intercultural [correo electrónico]. Dirección de Desarrollo Curricular del Ministerio de Educación Pública, San José, Costa Rica.

Duncan, Q. (2005). El negro en la Costa Rica de hoy. Revista del CESLA, (7), 399-404. 
Duncan, Q. y Meléndez, C. (2011). El negro en Costa Rica. San José: Editorial Costa Rica.

Fernández, L. (2006). ¿Cómo analizar datos cualitativos? Barcelona: Institut de Ciències de l'Educació, Universitat de Barcelona.

Flick, U. (2007). Introducción a la investigación cualitativa. 2da Ed. Madrid, España: Ediciones Morata..

García, N., y Martinell, A. (2010). El poder de la diversidad cultural (de XIII a XXI) [Presentación de diapositivas]. Recuperado de la plataforma Moodle de la Universidad Estatal a Distancia, Costa Rica.

Gomes, D. y Catchcart, M. L. (2011). Cabo verde: lengua, cultura e identidad y su relación con la educación. Ciencia en su PC, (4), 122-134. Recuperado de https://bit.ly/2QmLcxt

Greene, S. (2007). Entre lo indio, lo negro y lo incaico: The Spatial Hierarchies of Difference in Multiculural Peru. Journal of Latin American and Caribbean Anthropology, 12(2), 329-355

Harro, B. (2000). The cycle of socialization. En M. Adams, W. J. Blumenfeld, R. Castañeda, H. W. Hackman, M. L. Peters, y X. Zúñiga (Eds.). Readings for diversity and social justice: An anthology on racism, antisemitism, sexism, heterosexism, ableism, and classism (pp. 15-21). New York: Routledge.

Instituto Nacional de Estadística y Censos [INEC] y United Nations International Children's Emergency Fund [UNICEF]. (2014). Costa Rica: Indicadores de Educación y de Contexto. San José: UNICEF.

Instituto de Estudios Sociales en Población. (2009). Percepciones y actitudes de la población costarricense hacia la población Afrodescendiente. Heredia: Facultad de Ciencias Sociales de la Universidad Nacional.

Maldonado, N. (2007). On the Coloniality of Being: Contributions to the Development of a Concept, Cultural Studies, 21(2-3), 240-270.

Ministerio de Educación Pública de Costa Rica. (2009). Lo propio, lo nuestro, lo de todos. Educación e Interculturalidad. San José: Ministerio de Educación Pública de Costa Rica.

Ministerio de Educación Pública de Costa Rica. (2018). Departamento de Educación Intercultural. Recuperado de ht tp://www.ddc.mep.go.cr/educacion-intercultural

Ministerio de Educación Pública de Costa Rica. (2019). El Ministerio de Educación Pública. Recuperado de https:// www.mep.go.cr/

Molina, I. (2015). Costarricense por dicha: identidad nacional y cambio cultural en Costa Rica durante los siglos XIX y $X X$. San José: Editorial UCR.

Morlachetti, A. (2015). La Convención sobre los Derechos del Niño y la Protección de la Infancia en la Normativa Internacional de Derechos Humanos. Recuperado de https://bit.ly/2KeVzz8

Norton, B. (2000). Identity and Language Learning. Harlow: Longman.

Organización de las Naciones Unidas [ONU]. (1966). Pacto Internacional de Derechos Económicos, Sociales y Culturales. Recuperado de http://www.ohchr.org/SP/ProfessionalInterest/Pages/CESCR.aspx

Organización de las Naciones Unidas [ONU]. (1999). Aplicación del Pacto Internacional de los Derechos Económicos, Sociales y Culturales, Observación general 13, El derecho a la educación (artículo 13 del Pacto), (210 periodo de sesiones, 1999), U.N. Doc. E/C.12/1999/10 (1999). Recuperado de http://wwwl.umn.edu/humanrts/gencom $\mathrm{m} /$ epcomm 13s.htm

Pierce, B. N. (1995). Social Identity, investment and language learning. TESOL Quarterly, 29(1), 9-13.

Pizarro, G. (2003). Educación alternativa e identidad de grupos étnicos subordinados. Heredia: Universidad Nacional.

Programa de las Naciones Unidas para el Desarrollo [PNUD]. (2013a). Situación socioeconómica de la población afrodescendiente de Costa Rica según datos del X Censo Nacional de Población y VI de Vivienda 2011. Panamá: Programa de las Naciones Unidas para el Desarrollo.

Programa de las Naciones Unidas para el Desarrollo [PNUD]. (2013b). Informe nacional sobre desarrollo bumano 2013. San José: Programa de las Naciones Unidas para el Desarrollo.

Programa de las Naciones Unidas para el Desarrollo [PNUD]. (2017). Visión 2030. Poblaciones excluidas en Costa Rica. San José: Programa de las Naciones Unidas para el Desarrollo.

Quijano, A. (2000). Colonialidad del poder, eurocentrismo y América Latina. En E. Lander. (Comp.).La colonialidad del saber: eurocentrismo y ciencias sociales. Perspectivas latinoamericanas (pp. 777- 832).. Buenos Aires: CLACSO 
Racismo y matonismo en la escuela [Editorial]. (14 de febrero de 2012). La Nación. Recuperado de https://bit.ly/2 $\mathrm{QcBC} x 1$

Restrepo, E. (2002). Políticas de la alteridad: etnización de "comunidad negra" en el pacífico sur colombiano. Journal of Latin American Anthropology, 7(2), 34-58.

Singleton, G. (2015). Courageous conversations about race: A Field Guide for Achieving Equity in Schools (2 ${ }^{\mathrm{da}}$ Ed.). California: Corwin.

Suasnábar, C. (2013). La institucionalización de la educación como campo disciplinar. Investigación Educativa, 18(59), 1281-1304.

Suárez, N. (2007). La investigación documental paso a paso. Venezuela: Universidad de los Andes. Recuperado de h ttps://bit.ly/3ahRDrC

Tubino, F. (2005). La interculturalidad crítica como proyecto ético y político. Trabajo publicado en el Encuentro Continental de Educadores Agustinos, Lima, Perú. Recuperado de https://bit.ly/2ViE5Ip

United Nations International Children's Emergency Fund [UNICEF]. (2006). Observaciones Generales del Comité de los Derechos del Niño. Recuperado de http://www.unicef.org/ceecis/crcgencommes.pdf

Walsh, C. (2008). Interculturalidad crítica, pedagogía decolonial. En W. Villa y A. Grueso. (Comp.). Diversidad, interculturalidad y construcción de ciudad (pp. 1-18). Bogotá: Universidad Pedagógica Nacional/Alcaldía Mayor.

Walsh, C. (2009). Interculturalidad, Estado, Sociedad. Luchas (de) coloniales de nuestra época. Quito: Universidad Andina Simón Bolívar y Abya Yala.

Walsh, C. (2014). Afro In/Exclusion, Resistance, and the "Progressive" State. (De) colonial Struggles, Questions, and Reflections. En J. Muteba-Rahier (Ed.)., Black Social Movements in Latin America from Monocultural Mestizaje to Multiculturalism (pp. 15-34). New York, NY: Palgrave MacMillan.

Zimmer, T. (2011). El español hablado por los afrocostarricenses: Estudio lingüistico y sociolingüistico. Alemania: Kassel University Press.

CC BY-NC-ND 